\title{
CONVOLUTION THEOREMS WITH WEIGHTS
}

\author{
BY \\ R. A. KERMAN ${ }^{1}$
}

\begin{abstract}
Analogues of Young's Inequality and the Convolution Theorem are shown to hold when the $L_{p}$ and $L(p, q)$ spaces have underlying measure defined in terms of power weights.
\end{abstract}

I. Introduction. This paper deals with the convolution of two functions defined on $R^{n}$ :

$$
(f * g)(x)=\int_{R^{n}} f(x-y) g(y) d y, \quad x \in R^{n} .
$$

Well-known mapping properties of this positive, bilinear operator that involve the Lebesgue and Lorentz spaces are extended to the case in which these spaces have underlying measures defined by power weights.

Given a Lebesgue-measurable function $f$ on $R^{n}$, its distribution function with respect to the nonnegative locally integrable weight function $w(x)$ is

$$
\mu_{f}(s)=\int_{E_{s}} w(x) d x, \quad s>0,
$$

where $E_{s}=\left\{x \in R^{n}:|f(x)|>s\right\}$. The weighted Lorentz space $L(p, q ; w)$ consists of all Lebesgue-measurable functions on $R^{n}$ such that $\|f\|_{p, q ; w}$ is finite, with

$$
\begin{aligned}
& \|f\|_{p, q ; w}=\left[q \int_{0}^{\infty} s^{q-1} \mu_{f}(s)^{q / p} d s\right]^{1 / q}, \quad 1<p<\infty, 1 \leqslant q<\infty, \\
& \|f\|_{p, \infty ; w}=\sup _{s>0} s \mu_{f}(s)^{1 / p}, \quad 1 \leqslant p<\infty .
\end{aligned}
$$

For $p=q, L(p, p ; w)=L(p ; w)$, is a weighted Lebesgue space. Indeed, writing $\|f\|_{p, p ; w}$ as $\|f\|_{p ; w}$, we have

$$
\begin{aligned}
\|f\|_{p ; w}= & {\left[\int_{R^{n}} \mid f(x) p^{p} w(x) d x\right]^{1 / p}, \quad 1 \leqslant p<\infty, } \\
& \|f\|_{\infty ; w}=\underset{x \in R^{n}}{\operatorname{ess} \sup }|f(x) w(x)| .
\end{aligned}
$$

The spaces $L(p, q ; w)$ of interest to us have weights of the form $w(x)=|x|^{\alpha p}$, with $w(x)=|x|^{\alpha}$ in case $p=\infty$. In this context it is convenient to use the notations

Received by the editors August 23, 1982.

1980 Mathematics Subject Classification. Primary 42A35.

${ }^{1}$ Research partly supported by NSERC Grant \#A 4021 . 
$L(p, q ; \alpha), L(p ; \alpha),\|f\|_{p, q ; \alpha}$ and $\|f\|_{p ; \alpha}$. Finally, when $\alpha=0$, the case of Lebesgue measure, we write $L(p, q), L_{p},\|f\|_{p, q}$ and $\|f\|_{p}$. We mention here that the Lebesgue measure of the subset of $E$ of $R^{n}$ will be denoted by $|E|$.

Consistent use will be made of expressions of the form $X * Y \subset Z$ involving function spaces $X, Y$ and $Z$. These indicate that whenever $f \in X, g \in Y$, then $f * g \in Z$ with

$$
\|f * g\|_{Z} \leqslant K\|f\|_{X}\|g\|_{Y},
$$

the positive constant $K$ being independent of $f$ and $g$.

The classical result concerning convolution is

THEOREM 1.1 (Young's INEQUALITY).

$$
L_{p} * L_{q} \subset L_{r}, \quad 1<p, q, r<\infty,
$$

provided $1 / r=1 / p+1 / q-1$.

Our generalization of this, Theorem 3.1, states that

$$
L(p ; \alpha) * L(q ; \beta) \subset L(r ;-\gamma),
$$

given certain conditions, two of which are

$$
\frac{1}{r}=\frac{1}{p}+\frac{1}{q}+\frac{\alpha+\beta+\gamma}{n}-1 \text { and } \frac{1}{r} \leqslant \frac{1}{p}+\frac{1}{q} .
$$

We will refer to the first condition as the basic equality. More recently, O'Neil [7] has proved

\section{Theorem 1.2 (The Convolution Theorem).}

$$
L\left(p_{0}, q_{0}\right) * L\left(p_{1}, q_{1}\right) \subset L(p, q), \quad 1<p_{0}, p_{1}, p<\infty,
$$

provided

$$
\frac{1}{p}=\frac{1}{p_{0}}+\frac{1}{p_{1}}-1 \text { and } 0 \leqslant \frac{1}{q} \leqslant \frac{1}{q_{0}}+\frac{1}{q_{1}} \leqslant 1 .
$$

See also Hunt [4], Yap [11], and Blozinski [1].

Building on the Lebesgue case, we obtain, in Theorem 4.1, the extension

$$
L\left(p_{0}, q_{0} ; \alpha\right) * L\left(p_{1}, q_{1} ; \beta\right) \subset L(p, q ;-\gamma), \quad 1<p_{0}, p_{1}, p<\infty,
$$

where, among other restrictions,

$$
\frac{1}{p}=\frac{1}{p_{0}}+\frac{1}{q_{0}}+\frac{\alpha+\beta+\gamma}{n}-1 \text { and } 0 \leqslant \frac{1}{q} \leqslant \frac{1}{q_{0}}+\frac{1}{q_{1}} \leqslant 1 .
$$

Two proofs are given of a special case of this in $\$ 4$.

In the proof of Theorem 3.1, we require some properties of the fractional integration operator (or Riesz potential) $T_{\lambda}$, of order $\lambda$, defined at Lebesgue-measurable $f$ on $R^{n}$ by

$$
\left(T_{\lambda} f\right)(x)=\int_{R^{n}} \frac{f(y)}{|x-y|^{n-\lambda}} d y, \quad 0<\lambda<n,
$$


whenever the integral exists a.e. Stein and Weiss [8] extended to $T_{\lambda}$ the famous theorem of Hardy and Littlewood concerning fractional integration on the real line. For a simpler proof see [6]. With a slight change of notation, the extension reads

THEOREM 1.3. Let $0<\lambda<n, 1<p<\infty, \alpha<n(1-1 / p), \gamma<n / q, \alpha+\gamma \geqslant 0$ and $1 / q=1 / p+(\alpha+\gamma-\lambda) / n$. If $p \leqslant q<\infty$, then $T_{\lambda}: L(p ; \alpha) \rightarrow L(q ;-\gamma)$.

The case $\alpha=\gamma=0$ of Theorem 1.3 was obtained previously by Sobolev. O'Neil used the Convolution Theorem in [7] to prove a similar result for $L(p, q)$ spaces. As an application of Theorem 4.1, the analogue of O'Neil's theorem for power weights is given.

The author would like to thank Professor Richard Hunt for his contributions to this paper. In particular, the form of the proof of Proposition 4.2 and the essential idea behind the alternative proof of Theorem 4.1 are due to him.

II. Interpolation theorems. In this section we state, for easy reference, the interpolation theorems that will be used in our proofs. In these theorems, the primary indices, $p$, lie in $[1, \infty)$; the secondary indices, $q$, in $[1, \infty]$.

TheOREM 2.1 (WEAK-TYPE THEOREM). Suppose $T$ is a linear operator satisfying

$$
T: L\left(p_{i}, 1 ; w\right) \rightarrow L\left(p_{i}^{\prime}, \infty ; w^{\prime}\right)
$$

with operator norm $K_{i}, i=0,1 ; p_{0}<p_{1}, p_{0}^{\prime} \neq p_{1}^{\prime}$. Then,

$$
T: L\left(p_{t}, r ; w\right) \rightarrow L\left(p_{t}^{\prime}, r ; w^{\prime}\right), \quad 0<t<1,1 \leqslant r \leqslant \infty,
$$

where $1 / p_{t}=(1-t) / p_{0}+t / p_{1}, 1 / p_{t}^{\prime}=(1-t) / p_{0}^{\prime}+t / p_{1}^{\prime}$. Moreover, the operator norm is bounded by a constant (depending only on $t$ ) times $\max \left[K_{1}, K_{2}\right]$.

For a proof see [4].

REMARK 2.2. A linear operator satisfying the hypotheses of Theorem 2.1 is said to be of restricted weak-types $\left(p_{0}, p_{0}^{\prime}\right)$ and $\left(p_{1}, p_{1}^{\prime}\right)$. Stein and Weiss [10] showed that $T$ is of restricted weak-type $\left(p, p^{\prime}\right), p^{\prime}>1$, if and only if

$$
\int_{G} T \chi_{F}(x) w^{\prime}(x) d x \leqslant K\left[\int_{F} w(x) d x\right]^{1 / p}\left[\int_{G} w^{\prime}(x) d x\right]^{1-1 / p^{\prime}},
$$

the constant $K$ being independent of Lebesgue-measurable $F$ and $G$ of finite Lebesgue measure. It is known [4] that the bound of $T$ as an operator between $L(p, 1 ; w)$ and $L\left(p^{\prime}, \infty, w^{\prime}\right)$ is then at most $2 K$.

The next two results are special cases of a multilinear interpolation theorem proved in Calderon [2,10.2]; in the notation of that paper,

$$
\left[L\left(p_{0}, q_{0} ; w\right), L\left(p_{1}, q_{1} ; w\right)\right]_{t}=L\left(p_{t}, q_{t} ; w\right)
$$

and

$$
\left[L\left(p_{0} ; w_{0}\right), L\left(p_{1} ; w_{1}\right)\right]_{t}=L\left(p_{t} ; w_{t}\right)
$$

where

$$
\frac{1}{p_{t}}=\frac{1-t}{p_{0}}+\frac{t}{p_{1}}, \quad \frac{1}{q_{t}}=\frac{1-t}{q_{0}}+\frac{t}{q_{1}} \quad \text { and } \quad w_{t}=w_{0}^{p_{t}(1-t) / p_{0}} w_{1}^{p_{t} / p_{1}} .
$$


Theorem 2.3 (Strong-Type Multilinear Theorem). Let $T$ be a multilinear operator for which

$$
T: L\left(p_{i}, q_{i} ; w\right) \times L\left(p_{i}^{\prime}, q_{i}^{\prime} ; w^{\prime}\right) \rightarrow L\left(p_{i}^{\prime \prime}, q_{i}^{\prime \prime} ; w^{\prime \prime}\right)
$$

with norm $K_{i}, i=0,1$. Then,

$$
T: L\left(p_{t}, q_{t} ; w\right) \times L\left(p_{t}^{\prime}, q_{t}^{\prime} ; w^{\prime}\right) \rightarrow L\left(p_{t}^{\prime \prime}, q_{t}^{\prime \prime} ; w^{\prime \prime}\right),
$$

the norm being at most $K_{0}^{1-t} K_{1}^{t}$, where $p_{t}, p_{t}^{\prime}, p_{t}^{\prime \prime}, q_{t}, q_{t}^{\prime}$ and $q_{t}^{\prime \prime}$ are given as in (2.1).

Theorem 2.4 (Multilinear Change of Measure Theorem). Suppose $T$ is $a$ multilinear operator satisfying

$$
T: L\left(p_{i} ; w_{i}\right) \times L\left(p_{i}^{\prime} ; w_{i}^{\prime}\right) \rightarrow L\left(p_{i}^{\prime \prime} ; w_{i}^{\prime \prime}\right)
$$

with norm $K_{i}, i=0,1$. Then,

$$
T: L\left(p_{t} ; w_{t}\right) \times L\left(p_{t}^{\prime} ; w_{t}^{\prime}\right) \rightarrow L\left(p_{t}^{\prime \prime} ; w_{t}^{\prime \prime}\right)
$$

with norm at most $K_{0}^{1-t} K_{1}^{t}$, where $p_{t}, p_{t}^{\prime}, p_{t}^{\prime \prime}, w_{t}, w_{t}^{\prime}$ and $w_{t}^{\prime \prime}$ are given as in (2.1).

REMARK 2.5. In case $w_{i}(x)=|x|^{\alpha_{i} p_{i}}, w_{i}^{\prime}(x)=|x|^{\alpha_{i}^{\prime} p_{i}^{\prime}}$ and $w_{i}^{\prime \prime}(x)=|x|^{\alpha_{i}^{\prime \prime} p_{i}^{\prime \prime}}$, it is convenient to think of $\left(1 / p_{i}, 1 / p_{i}^{\prime}, 1 / p_{i}^{\prime \prime} ; \alpha_{i}, \alpha_{i}^{\prime}, \alpha_{i}^{\prime \prime}\right), i=0,1$, as endpoints of an interpolation interval and $t$ as the ratio in which the point $\left(1 / p_{t}, 1 / p_{t}^{\prime}, 1 / p_{t}^{\prime \prime} ; \alpha_{t}, \alpha_{t}^{\prime}, \alpha_{t}^{\prime \prime}\right)$ divides the interval. Here $\alpha_{t}=(1-t) \alpha_{0}+t \alpha_{1}$,

$$
\alpha_{t}^{\prime}=(1-t) \alpha_{0}^{\prime}+t \alpha_{1}^{\prime} \text { and } \alpha_{t}^{\prime \prime}=(1-t) \alpha_{0}^{\prime \prime}+t \alpha_{1}^{\prime \prime} \text {. }
$$

Recall that if, say, $p_{0}=\infty$, then $w_{0}(x)=|x|^{\alpha_{0}}$.

III. Convolution on the Lebesgue spaces. The purpose of this section is to prove THEOREM 3.1 .

$$
L(p ; \alpha) * L(q ; \beta) \subset L(r ;-\gamma)
$$

provided

(i) $1 / r=1 / p+1 / q+(\alpha+\beta+\gamma) / n-1,1<p, q, r<\infty, 1 / r \leqslant 1 / p+1 / q$,

(ii) $\alpha<n(1-1 / p), \beta<n(1-1 / q), \gamma<n / r$,

(iii) $\alpha+\beta, \beta+\gamma, \gamma+\alpha \geqslant 0$.

Some preliminary results will be required. To begin, we distinguish three cases.

(1) $\alpha, \beta>0 ; \gamma<0$,

(2) $\alpha<0 ; \beta, \gamma>0$ or $\beta<0$; $\alpha, \gamma>0$,

(3) $\alpha, \beta, \gamma \geqslant 0$.

The relation between these is seen in the following duality result.

Lemma 3.2. Suppose $a$ and $b$ are real numbers and $1<r \leqslant \infty, 1 \leqslant s<\infty$. Let $f$ be a nonnegative, Lebesgue-measurable function on $R^{n}$ and define the linear operator $T_{f}$ at Lebesgue-measurable $g$ by

$$
\left(T_{f} g\right)(x)=\int_{R^{n}} f(x-y) g(y) d y,
$$

whenever the integral exists for almost all $x$. Then,

$$
T_{f}: L(r ; a) \rightarrow L(s ; b),
$$


with operator norm $C$, implies

$$
T_{f}: L\left(s^{\prime} ;-b\right) \rightarrow L\left(r^{\prime} ;-a\right), \quad r^{\prime}=\frac{r}{r-1}, s^{\prime}=\frac{s}{s-1},
$$

with the same norm.

Proof. It is enough to show that given nonnegative $g \in L\left(s^{\prime} ;-b\right)$,

$$
\int_{R^{n}}\left(T_{f} g\right)(x) h(x)|x|^{-a r^{\prime}} d x \leqslant C\|g\|_{s^{\prime} ;-b},
$$

for all nonnegative $h$ satisfying

$$
h(x)|x|^{-a r^{\prime}} \in L(r ; a)
$$

with

$$
\left\|h(x)|x|^{-a r^{\prime}}\right\|_{r: a}=1 .
$$

Interchange of the order of integration in the integral in (3.4), then simple changes of variable, gives

$$
\int_{R^{\prime \prime}} g(-y) d y \int_{R^{\prime \prime}} f(y-x) h(-x)|x|^{-a r^{\prime}} d x .
$$

Hölder's inequality, with indices $s$ and $s^{\prime}$ corresponding to the functions

$$
|y|^{b} \int_{R^{n}} f(y-x) h(-x)|x|^{-a r^{\prime}} d x \text { and }|y|^{-b} g(-y),
$$

together with (3.2), now shows (3.3) to hold.

REMARK 3.3. We note here a number of consequences of the lemma that will be useful later. Numbered according to cases to which they refer, they are

(1) Theorem 3.1 holds in the first case if and only if it holds in either of the subcases of the second.

(2) It follows from consequence (1) that one need only consider the subcase in which $\alpha<0$. Given this, one has the theorem for $1 / r \leqslant 1 / p$ if and only if one has it for $1 / p+1 / q \geqslant 1$.

(3) The theorem is true for $1 / r \leqslant \max [1 / p, 1 / q]$ if and only if it is true for $1 / p+1 / q \geqslant 1$.

The next theorem, given in Okikiolu [6] in the unweighted case, is essential to proving our result when $1 / p+1 / q \geqslant 1$. Observe that, given the hypotheses, one has $\beta+\gamma, \gamma+\alpha>0$.

THEOREM 3.4. If, for $1<p, q<\infty$, we have

(i)

$$
2=\frac{1}{p}+\frac{1}{q}+\frac{\alpha+\beta+\gamma}{n}, \quad 1 \leqslant \frac{1}{p}+\frac{1}{q}
$$

(ii)

$$
\alpha<n(1-1 / p), \quad \beta<n(1-1 / q), \quad 0<\gamma<n,
$$

(iii)

$$
\alpha+\beta \geqslant 0,
$$


then

$$
L(p ; \alpha) * L(q ; \beta) \subset L(1 ;-\gamma)
$$

By duality, we obtain

$$
L(p ; \alpha) * L(\infty ; \beta) \subset L(r ;-\gamma)
$$

provided

(i)

$$
\frac{1}{r}=\frac{1}{p}+\frac{\alpha+\beta+\gamma}{n}-1, \quad \frac{1}{r} \leqslant \frac{1}{p},
$$

(ii)

$$
\alpha<n(1-1 / p), \quad 0<\beta<n, \quad \gamma<n / r,
$$

(iii)

$$
\alpha+\gamma \geqslant 0
$$

and

$$
L(\infty ; \alpha) * L(q ; \beta) \subset L(r ;-\gamma)
$$

provided

(i)

$$
\frac{1}{r}=\frac{1}{q}+\frac{\alpha+\beta+\gamma}{n}-1, \quad \frac{1}{r} \leqslant \frac{1}{q},
$$

(ii)

$$
0<\alpha<n, \quad \beta<n(1-1 / q), \quad \gamma<n / r,
$$

(iii)

$$
\beta+\gamma \geqslant 0 \text {. }
$$

Proof. Let $f$ and $g$ be nonnegative, Lebesgue-measurable functions on $R^{n}$. By Tonelli's theorem,

$$
\int_{R^{n}}(f * g)(x)|x|^{-\gamma} d x=\int_{R^{n}}\left(T_{n-\gamma} f\right)(x) g(-x) d x .
$$

Hölder's inequality applied to the functions

$$
\left(T_{n-\gamma} f\right)(x)|x|^{-\beta} \text { and } g(-x)|x|^{\beta}
$$

now gives

$$
\|f * g\|_{1 ;-\gamma} \leqslant K\left\|T_{n-\gamma} f\right\|_{q^{\prime} ;-\beta}\|g\|_{q ; \beta}, \quad q^{\prime}=\frac{q}{q-1} .
$$

Finally, the Stein-Weiss theorem on fractional integration implies, given our conditions,

$$
\left\|T_{n-\gamma} f\right\|_{q^{\prime} ;-\beta} \leqslant K\|f\|_{p ; \alpha} .
$$

Proof of TheOrem 3.1. In view of Remark 3.3, we may assume $\beta, \gamma \geqslant 0$. Two cases will be considered, depending on the size of $1 / p+1 / q$. 
Case I. $1 \leqslant 1 / p+1 / q$.

Theorem 2.4 will be used to interpolate between Hölder's inequality and (3.5) of Theorem 3.4. The endpoint corresponding to the classical result has $1 / p_{0}+1 / q_{0}=1$, $1 / r_{0}=0$; the other $1 / r_{1}=1$. Hence, the ratio in which the point of interest, $(1 / p, 1 / q, 1 / r ; \alpha, \beta, \gamma)$, divides the interval is $t=1 / r$. Further, we require $\alpha_{0}=\beta_{0}$ $=\gamma_{0}=0$. This entails $\alpha_{1}=\alpha r, \beta_{1}=\beta r, \gamma_{1}=\gamma r$, so, in particular, $0<\gamma_{1}<n$ and $\alpha_{1}+\beta_{1} \geqslant 0$. The remaining space parameters all depend on the choice of $1 / p_{0}$.

The condition $\beta_{1}<n\left(1-1 / q_{1}\right)$ is equivalent to

$$
\frac{1}{p_{0}}<\frac{(1-1 / q-\beta / n)}{(1-1 / r)} .
$$

If the right side of (3.8) is greater than 1 , that is $1 / r-1 / q>\beta / n$, take $1 / p_{0}=1$ and, hence, $1 / q_{0}=0,1 / p_{1}=r[1 / p+1 / r-1], 1 / q_{1}=r / q$. If $1 / r-1 / q \leqslant \beta / n$, take $1 / p_{0}=(1-1 / q-\beta / n) /(1-1 / r)$, which means

$$
\begin{gathered}
\frac{1}{q_{0}}=\frac{(1 / q+\beta / n-1 / r)}{(1-1 / r)}, \\
\frac{1}{p_{1}}=r\left[\frac{1}{p}+\frac{1}{q}-1+\frac{\beta}{n}\right]=r\left[\frac{1}{r}-\frac{(\alpha+\gamma)}{n}\right], \quad \frac{1}{q_{1}}=r\left[\frac{1}{r}-\frac{\beta}{n}\right] .
\end{gathered}
$$

Actually, in the latter instance one must choose $1 / p_{0}$ somewhat less than

$$
\frac{1-\frac{1}{q}-\frac{\beta}{n}}{1-\frac{1}{r}}
$$

Case II. $1 / p+1 / q<1$.

It follows from Case I and Remark 3.3 that (3.1) holds when

$$
1 / r \leqslant 1 / p
$$

or, indeed, if $\alpha \geqslant 0$, when

$$
1 / r \leqslant \max [1 / p, 1 / q]
$$

It may be supposed, then, that, at least,

$$
1 / p<1 / r \leqslant 1 / p+1 / q .
$$

We attempt to use Theorem 2.4 again, with the endpoints corresponding to (3.6) and (3.7). Thus, we set $1 / q_{0}=0,1 / r_{0}=1 / p_{0}$, and $1 / p_{1}=0$. The expression

$$
t=\frac{1 / p-1 / p_{0}}{1 / p_{1}-1 / p_{0}}
$$

simplifies to $1-1 / p$ if $1 / p_{0}=1$. Of course, $1 / p_{0}$ has to be taken less than 1 , though it will be convenient to keep $t=1-p_{0} / p$. Proceeding formally, one would have, in the ideal situation,

$$
\frac{1}{r_{0}}=\frac{1}{p_{0}}=1, \quad \frac{1}{q_{0}}=0, \quad \frac{1}{p_{1}}=0,
$$




$$
\frac{1}{q_{1}}=\frac{p^{\prime}}{q}, \quad \frac{1}{r_{1}}=\left(\frac{1}{r}-\frac{1}{p}\right) p^{\prime}, \quad p^{\prime}=\frac{p}{p-1} .
$$

Observe that $1 / r_{1} \leqslant 1 / q_{1}$.

For simplicity let $\alpha_{1}=0\left((3.7)\right.$ requires $\left.0<\alpha_{1}<n\right)$ and $\beta_{1}=n\left[1-1 / q_{1}\right]=$ $n(1-1 / p-1 / q) p^{\prime}$. Then

$$
\gamma_{1}=n\left(\frac{1}{r}-\frac{1}{p}\right) p^{\prime}, \quad \alpha_{0}=\alpha p_{1}, \quad \beta_{0}=n\left[\frac{1}{r}-\frac{\alpha+\gamma}{n}\right] p
$$

and

$$
\gamma_{0}=n[1-1 / q-(\alpha+\beta) / n] p .
$$

It can now be seen that both endpoints satisfy the conditions in the dual part of Theorem 3.4 when $\alpha<0$, that is, provided the indicated small adjustments are made in the above choices to ensure strict inequalities, such as $\beta_{1}<n\left[1-1 / q_{1}\right]$, hold when needed.

Finally, we must prove (3.1) when $\alpha>0$ and

$$
1 / q<1 / r \leqslant 1 / p+1 / q \text {. }
$$

In order that the first endpoint satisfy (3.6) we ask for $1 / r_{0}=1 / p_{0}$ and $1 / q_{0}=0$. The other one will come under (3.7) or (3.11) according as $1 / r=1 / p+1 / q$ or not. In either event, at the least, $1 / r_{1}=1 / q_{1}$ is necessary. Given this, $t=1-$ $p_{0}(1 / r-1 / q), 1 / p_{1}=(1 / t)(1 / p+1 / q-1 / r)$ and $1 / q_{1}=1 / q t$. We let $1 / p_{0}$ be slightly less than 1 .

All power indices can be determined from $\beta_{0}$ and $\gamma_{0}$. Thus, for example, by the basic equality, $\alpha_{0}=n-\left(\beta_{0}+\gamma_{0}\right)$. The constraints $\alpha_{0}<n\left(1-1 / p_{0}\right)$ and $\alpha_{1}<$ $n\left(1-1 / p_{1}\right)$ amount to $n<\beta_{0}+\gamma_{0}$ and

$$
\beta_{0}+\gamma_{0}<(\beta+\gamma) /(1-t) \text {. }
$$

We note that

$$
\beta+\gamma-n(1 / r-1 / q)=n(1-1 / p-\alpha / n)>0 .
$$

Again, $\beta_{0}<n\left(1-1 / q_{0}\right)$ and $\gamma_{0}<n / r_{0}$ will hold when

$$
\beta_{0}<n \text { and } \gamma_{0}<n \text {. }
$$

Inequalities (3.12) and (3.13) suggest choosing $\beta_{0}$ and $\gamma_{0}$ just smaller than $\min [n, \beta /(1-t)]$ and $\min [n, \gamma /(1-t)]$, respectively. The reader may easily verify that the resulting endpoints satisfy the appropriate conditions.

IV. Convolution on the Lorentz spaces. The results of the last section will be used to prove

\section{THEOREM 4.1.}

$$
L\left(p_{0}, q_{0} ; \alpha\right) * L\left(p_{1}, q_{1} ; \beta\right) \subset L(p, q ;-\gamma)
$$

provided

(i)

$$
\frac{1}{p}=\frac{1}{p_{0}}+\frac{1}{p_{1}}+\frac{\alpha+\beta+\gamma}{n}-1, \quad 1<p_{0}, p_{1}, p<\infty, 0 \leqslant \frac{1}{q} \leqslant \frac{1}{q_{0}}+\frac{1}{q_{1}} \leqslant 1,
$$


(ii)

$$
\alpha<n\left(1-\frac{1}{p_{0}}\right), \quad \beta<n\left(1-\frac{1}{p_{1}}\right), \quad \gamma<\frac{n}{p}
$$

(iii)

$$
\alpha+\beta, \quad \beta+\gamma, \quad \gamma+\alpha>0 .
$$

To proceed we must interpolate between weak-type estimates. These are given in

Proposition 4.2. Let $F, G$ and $H$ be subsets of $R^{n}$ having finite Lebesgue measure. Then,

$$
\begin{aligned}
& \int_{H}\left(\chi_{F}^{*} \chi_{G}\right)(x)|x|^{-\gamma p} d x \\
& \quad \leqslant K\left[\int_{F}|x|^{\alpha p_{0}} d x\right]^{1 / p_{0}}\left[\int_{G} \mid x \beta^{\beta p_{1}} d x\right]^{1 / p_{1}}\left[\int_{H}|x|^{-\gamma p} d x\right]^{1-1 / p}
\end{aligned}
$$

with $K$ independent of $F, G$ and $H$, provided

(i)

$$
\frac{1}{p}=\frac{1}{p_{0}}+\frac{1}{p_{1}}+\frac{\alpha+\beta+\gamma}{n}-1, \quad 1<p_{0}, p_{1}, p<\infty
$$

(ii)

$$
\alpha<n\left(1-\frac{1}{p_{0}}\right), \quad \beta<n\left(1-\frac{1}{p_{1}}\right), \quad \gamma<\frac{n}{p}
$$

(iii)

$$
\frac{1}{p_{0}}+\frac{1}{p_{1}}<1, \quad \operatorname{Max}\left[\frac{1}{p_{0}}, \frac{1}{p_{1}}\right]<\frac{1}{p} .
$$

Proof. We assume, for definiteness, that $\beta>0$. To start, write

$$
\left(\chi_{F} * \chi_{G}\right)(x)=\left(\int_{E_{1}}+\int_{E_{2}}+\int_{E_{3}}\right) \chi_{F}(y) \chi_{G}(x-y) d y=I_{1}(x)+I_{2}(x)+I_{3}(x)
$$

where

$$
E_{1}=\left\{y \in R^{n}:|y|>2|x|\right\}, \quad E_{2}=\left\{y \in R^{n}:|y|<2|x|,|x-y| \leqslant|x| / 2\right\},
$$

and

$$
E_{3}=\left\{y \in R^{n}:|y|<2|x|,|x-y|>|x| / 2\right\}
$$

Now,

$$
I_{1}(x) \leqslant 2 \int_{E_{1}} \chi_{F}(y)|y|^{\alpha} \chi_{G}(x-y)|x-y|^{\beta}|y|^{-\alpha-\beta} d y .
$$

Applying Hölder's inequality with indices $p_{0}, p_{1}$, and $s\left(1 / s=1-1 / p_{0}-1 / p_{1}\right)$ to the functions

$$
\chi_{F}(y)|y|^{\alpha}, \quad \chi_{G}(x-y)|x-y|^{\beta} \text { and } \chi_{E_{1}}(y)|y|^{-\alpha-\beta},
$$


respectively, we obtain $I_{1}(x)$ bounded by

$$
2\left[\int_{F}|x|^{\alpha p_{0}} d x\right]^{1 / p_{0}}\left[\int_{G}|x|^{\beta p_{1}} d x\right]^{1 / p_{1}}
$$

times

$$
\left[\int_{E_{1}}|y|^{-(\alpha+\beta) s} d y\right]^{1 / s} \leqslant K|x|^{\gamma-n / p}
$$

We claim

$$
\frac{\int_{H}|x|^{\gamma-n / p-\gamma p} d x}{\left[\int_{H}|x|^{-\gamma p} d x\right]^{1-1 / p}} \leqslant K
$$

Indeed, since $\gamma<n / p$, the ratio on the left side of (4.3) is smaller than the corresponding one in which $H$ is replaced by the sphere, $B_{\rho}$, of radius $\rho$ about the origin, with

$$
\int_{B_{\rho}}|x|^{-\gamma p} d x=\int_{H}|x|^{-\gamma p} d x
$$

But, this second ratio equals a constant times $c_{n}^{1 / p}$, where $c_{n}$ is the volume of the unit sphere in $R^{n}$.

From (4.3) it follows that (4.2) holds with $I_{1}(x)$ in place of $\left(\chi_{F} * \chi_{G}\right)(x)$.

A change of variable, followed by a reversal of the order of integration, gives

$$
\int_{H} I_{2}(x)|x|^{-\gamma p} d x=\int_{G} d z \int_{R^{n}} \chi_{F}(x-z) \chi_{H}(x)|x|^{-\gamma p} \chi_{E_{2}}(x-z) d x,
$$

the latter being no more than $2^{|\alpha|}$ times

$$
\int_{G} d z \int_{E_{4}} \chi_{F}(x-z)|x-z|^{\alpha} \chi_{H}(x)|x|^{\gamma-\gamma p}|x|^{-\alpha-\gamma} d x,
$$

where $E_{4}=\left\{x \in R^{n}:|x| \geqslant 2|z|\right\}$. Hölder's inequality with indices $p_{0}, p /(p-1)$ and $s, 1 / s=1 / p-1 / p_{0}$, shows $\int_{H} I_{2}(x)|x|^{-\gamma p} d x$ is dominated by

$$
\left[\int_{F}|x|^{\alpha p_{0}} d x\right]^{1 / p_{0}}\left[\int_{H}|x|^{-\gamma p} d x\right]^{1-1 / p} \int_{G}\left[\int_{E_{4}}|x|^{-(\alpha+\gamma) s} d x\right]^{1 / s} d z .
$$

However, the integral over $G$ in (4.4) is less than a constant multiple of

$$
\left.\int_{G}\left|x \beta^{\beta-n\left(1-1 / p_{1}\right)} d x \leqslant \int_{B_{\rho}}\right| x\right|^{\beta-n\left(1-1 / p_{1}\right)} d x \leqslant K\left[\int_{G}|x|^{\beta p_{1}} d x\right]^{1 / p_{1}},
$$

where $B_{\rho}$ is the sphere of radius $\rho$ about the origin for which

$$
\int_{B_{\rho}}|x|^{\beta p_{1}} d x=\int_{G}|x|^{\beta p_{1}} d x
$$

When $\alpha<0, I_{3}(x)$ may be treated in the same way as $I_{1}(x)$. In case $\alpha>0$, we must repeat, with $I_{3}(x)$, the argument given above for $I_{2}(x)$, assigning the roles of $F$ and $p_{0}$ to $G$ and $p_{1}$. 
REMARK 4.3. The conditions $\alpha+\beta, \beta+\gamma, \gamma+\alpha>0$, are automatically satisfied, given the assumptions of the above proposition.

Proof of Theorem 4.1. In view of Proposition 4.2 and Theorem 3.1, (4.2) always holds under our assumptions. It follows from Remark 2.2 that the operator $\chi_{F} \rightarrow$ $\chi_{F} * \chi_{G}$ can be extended to a bounded operator from $L\left(p_{0}, 1 ; \alpha\right)$ to $L(p, \infty ;-\gamma)$ with norm essentially equal to

$$
\left[\int_{G} \mid x \beta^{\beta p_{1}} d x\right]^{1 / p_{1}} .
$$

Fix $f \in L\left(p_{0}, 1 ; \alpha\right)$, as well as $p_{0}, \alpha, \beta p_{1}^{\prime} \equiv c$ and $-\gamma p^{\prime} \equiv k$, and consider the line $L$ defined by the relationship

$$
\frac{1+k / n}{p^{\prime}}=\frac{1}{p_{0}}+\frac{1+c / n}{p_{1}^{\prime}}+\frac{\alpha}{n}-1
$$

in the $\left(1 / p_{1}^{\prime}, 1 / p^{\prime}\right)$-plane. The mapping $\chi_{G} \rightarrow f * \chi_{G}$ satisfies restricted weak-type conditions for points on $L$ near $\left(1 / p_{1}, 1 / p\right)$, the weak-type norm in each case being bounded by a constant multiple of $\|f\|_{p_{0}, 1 ; \alpha}$. Theorem 2.1 thus yields

$$
L\left(p_{0}, 1 ; \alpha\right) * L\left(p_{1}, r ; \beta\right) \subset L(p, r ;-\gamma), \quad 1 \leqslant r \leqslant \infty .
$$

Next, take $g \in L\left(p_{1}, \infty ; \beta\right)$. Hold $p_{1}, \beta, \alpha p_{0}^{\prime} \equiv c$, and $-\gamma p^{\prime} \equiv k$ constant. Working with the mapping $f \rightarrow f * g$ and the equation

$$
\frac{1+k / n}{p^{\prime}}=\frac{1+c / n}{p_{0}^{\prime}}+\frac{1}{p_{1}}+\frac{\beta}{n}-1,
$$

we obtain

$$
L\left(p_{0}, r ; \alpha\right) * L\left(p_{1}, \infty ; \beta\right) \subset L(p, r ;-\gamma), \quad 1 \leqslant r \leqslant \infty .
$$

Similarly,

$$
L\left(p_{0}, \infty ; \alpha\right) * L\left(p_{1}, r ; \beta\right) \subset L(p, r ;-\gamma), \quad 1 \leqslant r \leqslant \infty .
$$

Taking $r=1$ in (4.6) and (4.7), the multilinear strong-type interpolation theorem, with $t=1 / s$, gives

$$
L\left(p_{0}, r ; \alpha\right) * L\left(p_{1}, s ; \beta\right) \subset L(p, 1 ;-\gamma),
$$

$1 / r+1 / s=1,1 \leqslant r \leqslant \infty$. The same theorem, applied to (4.6) and (4.8) with $r=q_{0}, t=q_{0} / q_{1}\left(q_{0}-1\right)$, yields

$$
L\left(p_{0}, q_{0} ; \boldsymbol{\alpha}\right) * L\left(p_{1}, q_{1} ; \beta\right) \subset L(p, q ;-\gamma)
$$

for $1 / q=1 / q_{0}+1 / q_{1}$ and hence, using the fact that $L(p, q ;-\gamma)$ increases with $q$, for $1 / q \leqslant 1 / q_{0}+1 / q_{1}$.

When $\alpha, \beta, \gamma \geqslant 0$, Theorem 4.1 can be proved using the Convolution Theorem. The conclusion of the latter is true without the restriction $1 / q_{0}+1 / q_{1} \leqslant 1$ (i.e., one only requires $\left.0<q_{0}, q_{1}<\infty\right)$, as shown in [1], and hence, the same will be so for the weighted case. This version of Theorem 4.1 implies Theorem 3.1.

Alternative Proof of Theorem 4.1 when $\alpha, \beta, \gamma \geqslant 0$. For $q<\infty$, we have

$$
\|f * g\|_{p, q ;-\gamma}=\left[q \int_{0}^{\infty} s^{q-1} \mu_{f * g}(s)^{q / p} d s\right]^{1 / q},
$$


where

$$
\mu_{f * g}(s)=\int_{E_{s}}|x|^{-\gamma p} d x, \quad E_{s}=\left\{x \in R^{n}:(f * g)(x)>s\right\} .
$$

The simple estimate

$$
\int_{E_{s}}|x|^{-\gamma p} d x \leqslant K\left|E_{s}\right|^{1-\gamma p / n}
$$

yields

$$
\|f * g\|_{p, q ;-\gamma} \leqslant K\|f * g\|_{p^{\prime}, q}, \quad 1 / p^{\prime}=1 / p-\gamma / n .
$$

By the Convolution Theorem, the term on the right side of (4.10) is smaller than a constant multiple of $\|f\|_{p_{0}^{\prime}, q_{0}}\|g\|_{p_{1}^{\prime}, q_{1}}$ with

$$
1 / p_{0}^{\prime}=1 / p_{0}+\alpha / n, \quad 1 / p_{1}^{\prime}=1 / p_{1}+\beta / n .
$$

From the elementary inequalities

$$
|E|^{1 / p_{0}^{\prime}} \leqslant K\left[\int_{E}|x|^{\alpha p_{0}} d x\right]^{1 / p_{0}}, \quad|E|^{1 / p_{1}^{\prime}} \leqslant K\left[\int_{E}|x|^{\beta p_{1}} d x\right]^{1 / p_{1}},
$$

which hold for any Lebesgue-measurable set $E$, it now follows that

$$
\|f * g\|_{p, q ;-\gamma} \leqslant K\|f\|_{p_{0}, q_{0} ; \alpha}\|g\|_{p_{1}, q_{1} ; \beta} .
$$

REMARK 4.4. The alternative proof does not require strict inequality in condition (iii) of Theorem 4.1.

As an application of Theorem 4.1, we obtain weighted Lorentz space inequalities for fractional integrals.

TheOREM 4.5. Suppose $0<\lambda<n, 1<p_{0}<\infty, \alpha<n\left(1-1 / p_{0}\right), \gamma<n / p, \alpha+\gamma$ $>0$, and $1 / p=1 / p_{0}+(\alpha+\gamma-\lambda) / n$. Then,

$$
T_{\lambda}: L\left(p_{0}, q_{0} ; \alpha\right) \rightarrow L(p, q ;-\gamma)
$$

for $q \geqslant q_{0}$.

Proof. The kernel $1 /|x|^{n-\lambda}$ of $T_{\lambda}$ can belong to a space $L\left(p_{1}, q_{1} ; \beta\right)$ only if $q_{1}=\infty$, and then, only with $p_{1}=n /(n-\beta-\lambda)$. This requires $0<\beta+\lambda<n$. We must choose $\beta$ satisfying this condition as well as $\beta<n\left(1-1 / p_{1}\right), \alpha+\beta>0$ and $\beta+\gamma>0$. If $\alpha, \gamma>0$, take $\beta$ to be a sufficiently small positive number; if $\alpha \leqslant 0$, take $\beta$ somewhat larger than $-\alpha$; if $\gamma<0$, take $\beta$ just bigger than $-\gamma$.

\section{REFERENCES}

1. A. P. Blozinski, On a convolution theorem for $L(p, q)$ spaces, Trans. Amer. Math. Soc. 164 (1972), 255-265.

2. A. P. Calderón, Intermediate spaces and interpolation, the complex method, Studia Math. 24 (1964), $113-190$.

3. G. H. Hardy, J. E. Littlewood and G. Polya, Inequalities, Cambridge Univ. Press, London, 1934.

4. R. A. Hunt, On $L(p, q)$ spaces, Enseign. Math. (2) 12 (1966), 249-276.

5. G. G. Lorentz, Some new functional spaces, Ann. of Math. (2) 51 (1950), 37-55.

6. G. O. Okikiolu, Aspects of the theory of bounded integral operators on $L^{p}$-spaces, Academic Press, New York, 1970. 
7. R. O'Neil, Convolution operators and $L(p, q)$ spaces, Duke Math. J. 30 (1963), 129-142.

8. E. M. Stein and G. Weiss, Fractional integrals on n-dimensional Euclidean space, J. Math. Mech. 7 (1958), 503-514.

9. __ Interpolation of operators with change of measures, Trans. Amer. Math. Soc. 87 (1958), $159-172$.

10. An extension of a theorem of Marcinkiewicz and some of its applications, J. Math. Mech. 8 (1959), 263-284.

11. L. Y. H. Yap, Some remarks on convolution operators and $L(p, q)$ spaces, Duke Math. J. 36 (1969), $647-658$.

Department of Mathematics, Brock University, St. Catharines, Ontario, Canada L2S 3A1 